\title{
Context effects in visual length perception: Role of ocular, retinal, and spatial location
}

\author{
YOAV ARIEH and LAWRENCE E. MARKS \\ John B. Pierce Laboratory, New Haven, Connecticut \\ and Yale University, New Haven, Connecticut
}

\begin{abstract}
In three experiments, we examined the transfer of orientation-contingent context effects between the eyes and across portions of the retina with or without variation in external spatial location. Previous research had shown that vertical lines are judged long, relative to horizontal lines, when the stimulus set comprises relatively long horizontals and short verticals (Contextual Condition B), as compared with the reverse when the stimulus set comprises relatively short horizontals and long verticals (Contextual Condition A). Consequently, the contextual set of stimuli influences the magnitude of the horizontal-vertical illusion (HVI), decreasing its size under Contextual Condition A and increasing its size under Contextual Condition B. Experiment 1 showed that exposing one eye to different stimulus contexts modulated the size of the HVI at the exposed eye but had little or no effect at the other eye. Experiments 2 and 3 showed that the effect of the contextual sets generalized poorly across adjacent portions of the retina but transferred almost perfectly across different locations in external space when retinal location was constant. Thus, orientation-contingent context effects in visual length perception appear to be specific to the eye and to the region of the retina stimulated, suggesting that these effects reflect relatively early and local changes in sensitivity, rather than relatively late and general shifts in response criteria.
\end{abstract}

Perceptual systems can adjust their sensitivity in response to preceding or ongoing stimulation. This capacity is evident in various phenomena called sensory fatigue or sensory adaptation (Canevet, Scharf, \& Botte, 1985; Meiselman, 1968; Pryor, Steinmetz, \& Stone, 1970; Verrillo \& Schmiedt, 1974). For example, prolonged and intense auditory or visual stimulation depresses the ability to detect subsequent threshold-level sounds or lights and attenuates the perceived intensity of subsequent signals of moderate intensity (Scharf, 1983; J. C. Stevens \& S. S. Stevens, 1963; Ward, 1963).

In recent years, adjustments in suprathreshold sensitivity have been reported under conditions in which sensory adaptation would not be expected. Using various psychophysical procedures in several modalities, especially hearing, Marks and colleagues have uncovered evidence that moderate, transient stimulation can induce perceptual systems to "recalibrate" their relative suprathreshold responsiveness (Marks, 1988; see also Marks, 1992b, 1994; Marks \& Warner, 1991). Consider the following example, taken from hearing. In one condition

This study was supported by Grant DC03842 from the National Institute of Deafness and Other Communicative Disorders, NIH, to the second author. We thank Carol Cicerone and two anonymous reviewers for their comments on an earlier version of the paper. Their suggestions led to the addition of Experiment 3, and for these suggestions we are especially grateful. Please address correspondence to Y. Arieh, John B. Pierce Laboratory, 290 Congress Avenue, New Haven, CT 06519 (e-mail: yarieh@jbpierce.org).
(A), participants were presented with a series of pure tones and made loudness judgments of each tone, using magnitude estimation. The series comprised $500-\mathrm{Hz}$ signals of relatively low sound pressure level (SPL) and $2500-\mathrm{Hz}$ signals of relatively high SPL. Note that the signals ranged overall in level from 30 to $85 \mathrm{~dB}$ and each lasted for only $1 \mathrm{sec}$, conditions that should not encourage shifts in sensitivity. In another condition (B), the $500-\mathrm{Hz}$ signals took on relatively high SPLs, and the $2500-\mathrm{Hz}$ signals took on relatively low SPLs. The judgments of loudness shifted substantially across the two conditions: In Condition A, a $500-\mathrm{Hz}$ tone at $70 \mathrm{~dB}$ was judged to be as loud as a $2500-\mathrm{Hz}$ tone at $73 \mathrm{~dB}$, whereas in Condition B the same $500-\mathrm{Hz}$ tone at $70 \mathrm{~dB}$ was judged to be as loud as a $2500-\mathrm{Hz}$ tone at $57 \mathrm{~dB}$. Thus, implicit loudness matches shifted across the two contextual conditions by $16 \mathrm{~dB}$ (Marks, 1988). These loudness shifts were dubbed differential context effects (DCEs), since loudness judgments at the two frequencies were differentially affected by experimental context (see also Marks, 1992b).

In Figure 1, we depict, in an idealized manner, the stimulus conditions that typically produce DCEs. Each contextual condition contains stimuli that act on two processing channels (e.g., frequencies of 500 and $2500 \mathrm{~Hz}$ in hearing) at several intensity levels. In Context $\mathrm{A}$, the average intensity level at Channel 2 is higher than the average level at Channel 1. Consequently, at the same physical intensity, the stimuli activating Channel 2 are judged to be weaker than the stimuli activating Channel 1. In 


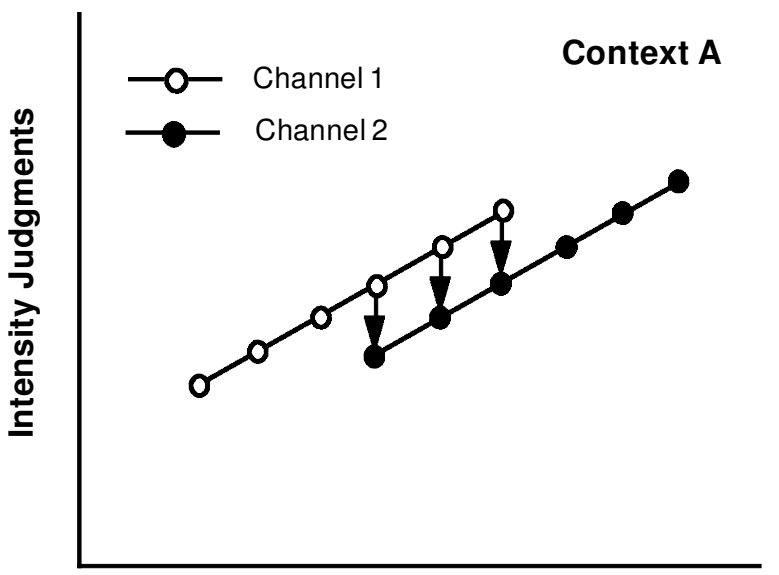

Intensity

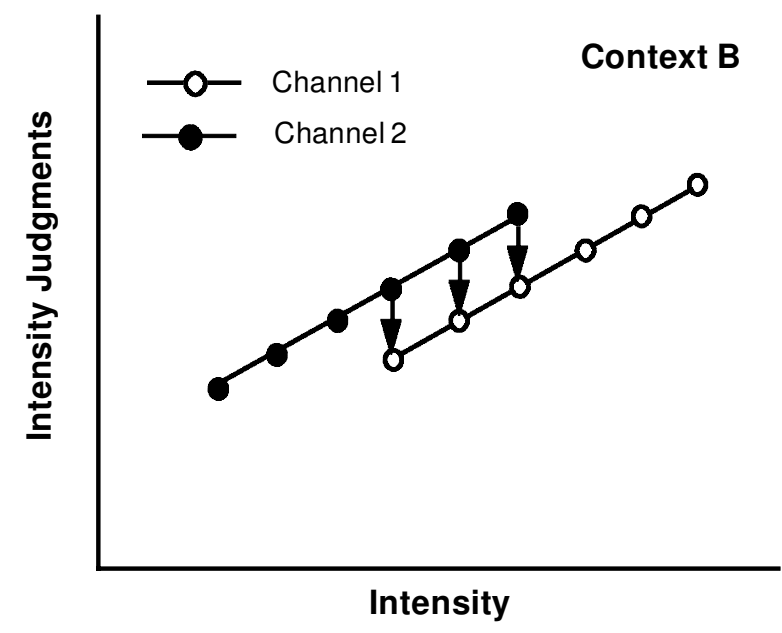

Figure 1. A schematic diagram of the differential context effects. In Context A, stimuli applied to Channel 2 have a higher average intensity than do stimuli applied to Channel 1, whereas in Context B, stimuli applied to Channel 1 have the higher average intensity. The overall shifts in judgments across the two contexts of stimuli common to both conditions (indicated by arrows) constitute the differential context effects.

Context B, the tables are turned. Now, the average intensity level at Channel 1 is higher than the average level at Channel 2. Accordingly, intensity judgments shift so that stimuli of the same physical intensity are judged to be weaker when they activate Channel 1 . The overall shift in intensity judgments in the two channels across the two contextual conditions constitutes the DCE.

Variations in stimulus context produce analogous results in other modalities, including taste, haptic touch, and vision. Thus, judgments of taste intensity of sucrose and $\mathrm{NaCl}$ shifted relative to each other when the average concentrations of the two tastants shifted (Rankin \& Marks, 1991), and the perceived extent of objects presented in radial and tangential orientations to the torso, felt by actively moving the hand, shifted when the aver- age lengths at the two orientations shifted (Marks \& Armstrong, 1996). Especially pertinent to the present study are the findings of Armstrong and Marks (1997), who reported that the perceived lengths of lines presented in horizontal and vertical orientations shifted when the mean physical lengths in the different orientations shifted. Thus, presenting longer vertical than horizontal lines caused the verticals to be underestimated, whereas presenting longer horizontal than vertical lines caused the horizontals to be underestimated (see also Potts, 1991).

Two essential and abiding questions about DCEs remain to be fully answered. First, where in the stream of perceptual processes are DCEs introduced, relatively early and peripherally, or later and more centrally? Second, what is it that is modified? Do the changes in the judgments of length reflect modifications in the underlying sensory representations of length at one orientation or both? Or do the changes reflect modifications in the decisional processes that participants use in judging and comparing stimuli? These questions are related, in that early, peripheral effects would almost certainly be sensory in nature, whereas later, more central effects might be sensory but could also reflect postsensory decisional processes. The present study speaks directly to this question as it applies to the visual perception of length of vertical and horizontal line segments. By trying to establish whether the shifts in responsiveness caused by DCEs are spatially specific, we hoped to shed some light on their nature.

DCEs were first observed in magnitude estimates of loudness, where Marks (1988) suggested a decisional interpretation: that DCEs represent frequency-specific numerical response biases in magnitude estimation. This is to say, participants might tend to apply a constant range of numbers to a given kind of stimulus even when the mean levels shift across contextual conditions. As a consequence, a signal of fixed SPL at a given frequency would receive a greater loudness judgment when the mean SPL at that frequency is low rather than high. The results of subsequent studies cast doubt on this interpretation, since DCEs have also been reported when participants did not make numerical judgments. Schneider and Parker (1990) had participants compare differences in the loudness of various pairs of tones while the average SPL at both frequencies shifted across conditions. Mapes-Riordan and Yost (1999) had participants compare loudness directly after presenting a brief recalibrating tone. The comparisons of loudness differences and of loudness revealed DCEs like those observed with magnitude estimation.

Although a numerical response model seems inadequate to explain DCEs in a difference comparison paradigm, DCEs nevertheless might result from a more general decisional/criterial process. Perhaps exposure to contextual stimuli causes response criteria to shift differentially at two frequencies so as to change judgments of relative loudness. According to this sort of model, lis- 
tening to relatively weak signals at Frequency 1 and relatively strong signals at Frequency 2 would shift response criteria so that a greater SPL would be needed at Frequency 2 to match the loudness of a given stimulus at Frequency 1 . Such a model could explain the shifts in judgment observed when mean SPLs of the frequencies vary across different contextual conditions. But even though it fares better than the numerical bias model, this broader decisional model also seems inconsistent with several experimental findings.

If DCEs reflect nothing more than shifts in decision criteria, independent of the sensory representations of the stimuli, one would expect the decisional process to be a general one-that is, a process that should characterize judgments in most (if not all) sense modalities. Although DCEs have been reported in several modalities, including taste (Rankin \& Marks, 1991, 1992), olfaction (Rankin \& Marks, 2000), haptic touch (Marks \& Armstrong, 1996), and vision (Armstrong \& Marks, 1997; Potts, 1991), DCEs were conspicuously absent from judgments of the duration of sound signals differing in frequency and from judgments of the length of lines differing in color (Marks, 1992b). These results are difficult to reconcile with a general decisional or criterial model, which should apply more universally.

According to the criterial model, DCEs arise when people apply different decisional rules to stimuli that appear perceptually distinct or dissimilar (e.g., different sound frequencies, different orientations of lines, different taste qualities); if so, DCEs should arise when stimuli are perceived as dissimilar, but not when they are perceived as similar. (In the limit, when the difference between the two stimuli becomes negligibly small, the two subcontexts merge into one.) Rankin and Marks (2000) had participants judge chemosensory stimuli whose similarity or dissimilarity was, in some cases, independent of the receptors (gustatory or olfactory) that were activated. That is, similarity could be dissociated from communality of sensory processing. The results showed substantial DCEs whenever different receptors were stimulated, even when the stimuli were judged to be qualitatively similar, leading Rankin and Marks to conclude that DCEs probably do not reflect the application of different response criteria to perceptually dissimilar stimuli.

The present study used a different approach to help illuminate the processes underlying DCEs, by asking whether changes in perceived length transfer from one eye to the other, from one retinal location to nearby locations, and from one region of the external space to adjacent regions of space. If DCEs are spatially specific, they will be evident only in the locations at which they are induced; DCEs should not transfer between the eyes, between different portions of the retina, or between different regions of external space. In many cases, determining whether a process is spatially specific provides a hint as to its nature. Thus, a process showing spatial specificity might reside relatively early in the perceptual stream and likely would involve changes in sensitivity, whereas a process showing spatial generalization might reside relatively late in the perceptual stream and might, although it need not, reflect the operation of a decisional mechanism.

A prototype for stimulus-induced changes in sensitivity is sensory adaptation. Sensory adaptation is often found to be spatially specific. Thus, poststimulatory shifts in auditory thresholds are largely restricted to the ear receiving stimulation and do not transfer to the contralateral ear (McPherson \& Anderson, 1971). Similarly, adapting one eye to darkness does not affect threshold or brightness judgments in the contralateral eye (J. C. Stevens \& S. S. Stevens, 1963). If the same were true for DCEs, which are produced by relatively moderate, transient stimulation, one would not expect to find any transfer in hearing between the two ears or in vision between the two eyes. Marks (1996) showed that exposing one ear to contextually varying sounds led to substantial loudness shifts in the exposed ear and to more modest shifts in the contralateral ear. This partial transfer implies a central mechanism that partially pools the sensitivity shifts across the two ears.

Note that DCEs in hearing also exhibit fatigue-like properties that are consistent with a peripheral origin. First, DCEs seem to depend largely on signals at relatively high SPL (Marks, 1993). That is, the changes in perceived loudness at a given SPL seem to stem mainly from some kind of attenuation brought about at the frequency at which the signals are relatively louder, rather than from amplification brought about at the frequency at which they are relatively softer. Second, the magnitude of the DCEs depends crucially on the difference between the two frequencies, being large when the difference is large but small or negligible when the difference falls roughly below a critical band (Marks, 1994; Marks \& Warner, 1991).

The present study extends Marks's (1996) interaural transfer paradigm to the modality of vision. DCEs in vision were first demonstrated in the perception of line length (Armstrong \& Marks, 1997; Marks \& Armstrong, 1996; Potts, 1991). Armstrong and Marks had participants made paired-comparisons judgments of the lengths of horizontal and vertical line segments. The resulting judgments of relative length depended systematically on the set of stimulus magnitudes. A given vertical line was judged to be shorter (i.e., matched a shorter horizontal line) when the stimulus set comprised relatively long verticals and short horizontals (Context $\mathrm{A}$ ) than when the stimulus set comprised relatively long horizontals and short verticals (Context B). The reverse was true for a given horizontal line, which was judged to be longer (i.e., matched a longer vertical line) under Context A than under Context B. Consequently, to the extent that stimulus context exerted orientation-specific effects on perceived length, it also modulated the magnitude of the horizontal-vertical illusion (HVI). Thus, relative to about 
$10 \%$ at baseline, the HVI shrank to $3 \%$ under Contextual Condition A (the verticals lines being underestimated) but ballooned to $15 \%$ under Contextual Condition B (the horizontal lines being underestimated). Thus, the magnitude of the HVI can serve as a useful indicator of the magnitude of the DCEs in judgments of line lengths at different orientations. Given the presence of DCEs, the HVI should decrease under Contextual Condition A and increase under Contextual Condition B.

In the experiments reported here, it was asked whether DCEs in judgments of line length transfer between the two eyes, between nearby regions of the retina, and between regions of external space. First, we asked whether exposing one eye to DCE-inducing sets of vertical and horizontal lines leads to changes in the relative magnitude of the HVI in the contralateral eye. Second, we asked whether exposing a selected portion of the retina to DCE-inducing sets of vertical and horizontal lines leads to changes in the relative magnitude of the HVI in adjacent regions of the retina. And third, we asked whether the results observed in Experiment 2 were due to the contextual sets' being presented to different regions of the retina or to different regions of external space. Failure to find interocular and interretinal transfer would not necessarily mean that DCEs reside wholly in an early visual process, but it would constitute supporting evidence. Furthermore, the finding that the DCEs are spatially specific would not be easily reconcilable with a general decisional model. Such a model predicts a general shift in response criteria that would not be limited to specific parts of the retina. On the other hand, the presence of interocular and interretinal transfer would suggest a central component to the mediation of DCE, although it would not indicate whether that component is sensory or decisional.

\section{EXPERIMENT 1A Baseline Measures}

Experiment 1A provided baseline measures of the HVI in the left and right eyes. We asked participants to judge, by magnitude estimation, the perceived length of horizontal and vertical line segments presented exclusively to one eye or the other. We expected that the vertical lines would be judged longer than physically equal horizontal lines (HVI). We also expected that the size of the HVI would lie between $9 \%$ and $15 \%$, a range reported in earlier studies (Armstrong \& Marks, 1997; Prinzmetal \& Gettleman, 1993). Finally, we had no compelling reason to expect that the size of the HVI would differ in the two eyes.

\section{Method}

Participants. All the participants in this and the following experiments were 18-40 years old and were recruited from the Yale community. Twelve participants, 4 men and 8 women, were paid to participate in Experiment $1 \mathrm{~A}$.

Apparatus. The participants were tested individually in a dark, sound-attenuated booth. The stimuli were presented on a 13-in.
Apple color monitor driven by a Power Macintosh equipped with a built-in 6100 video card. Screen resolution was $640 \times 480$ pixels. The frame of the monitor and the outer portion of the screen were masked by a $34.5 \times 34.5 \mathrm{~cm}$ matte black board containing an $18.5 \times$ $18.5 \mathrm{~cm}$ aperture at its center, through which a square central region on the screen was visible. Viewing distance was approximately $60 \mathrm{~cm}$ and was controlled by a head/chin-rest. From its center, a black, wooden board extended to the center of the screen, effectively bisecting the visible square on the screen and thereby making it possible to present stimuli exclusively to one eye or the other. A program written in PsyScope (Cohen, MacWhinney, Flatt, \& Provost, 1993) was used for stimulus presentation and data collection.

Stimuli and Procedure. Eight line segments, four at each orientation, made up the baseline set of stimuli. Their lengths were 0.95 , $1.9,2.85$, and $4.1 \mathrm{~cm}$. Lines were black against a very light gray background (luminance of $51 \mathrm{~cd} / \mathrm{m}^{2}$ for the screen and of $0.685 \mathrm{~cd} / \mathrm{m}^{2}$ for the surrounding black frame) and were 1 pixel thick. The location of each line on the screen was defined by its center, which always fell on the midpoint of one of the screen's halves.

Each trial began with a fixation point presented to the left or the right side of the screen. After a 300-msec delay, a line was presented for $500 \mathrm{msec}$. Subsequently, the participants were prompted to enter their judgments of the line length, using the computer keyboard. The method was magnitude estimation with no designated standard or modulus. To the first line, the participants were asked to assign whatever number seemed appropriate to stand for its length. Then, to succeeding stimuli, the participants were to assign numbers in proportion to the length, using whole numbers or decimals as needed. Pressing the enter key recorded the participant's response and initiated the next trial after an intertrial interval of $500 \mathrm{msec}$. Order of presentation was randomized so that each participant received a different sequence of stimuli. Following 10 practice trials, each line was presented six times to each eye, making 96 test trials overall. A typical session lasted about $15 \mathrm{~min}$.

\section{Results and Discussion}

In the following analysis and subsequent ones, numerical estimates were averaged arithmetically across replicates within subjects and geometrically across subjects. The baseline measures obtained for the left and the right eyes are presented in Figure 2. Two features are notable. First, the vertical lines were judged to be longer than the horizontal lines in both eyes. Second, the magnitude of the HVI seems comparable at both eyes. The computed magnitude of the HVI- $\left[100 *\left(J_{\mathrm{v}}-J_{\mathrm{h}}\right)\right] / J_{\mathrm{h}}-$ was $10.9 \%$ at the right eye and $12.0 \%$ at the left eye. The data (log transformed) were subjected to an analysis of variance (ANOVA), using exposed eye (left, right), orientation (horizontal, vertical), and length as within-subjects variables. The results verified the visual inspection of the data. Only two terms were significant: the expected main effects of length $[F(3,33)=316.3, p<.001]$ and orientation $[F(1,11)=203.4, p<.001]$. Given that the magnitudes of the HVI at the left and the right eyes were comparable, we used their average $(11.45 \%)$ as a yardstick to assess the magnitude of the interocular transfer in Experiment 1B.

\section{EXPERIMENT 1B Interocular Transfer of the DCE}

In Experiment 1B, we tested whether a DCE induced in one eye can modulate the size of the HVI in the other 


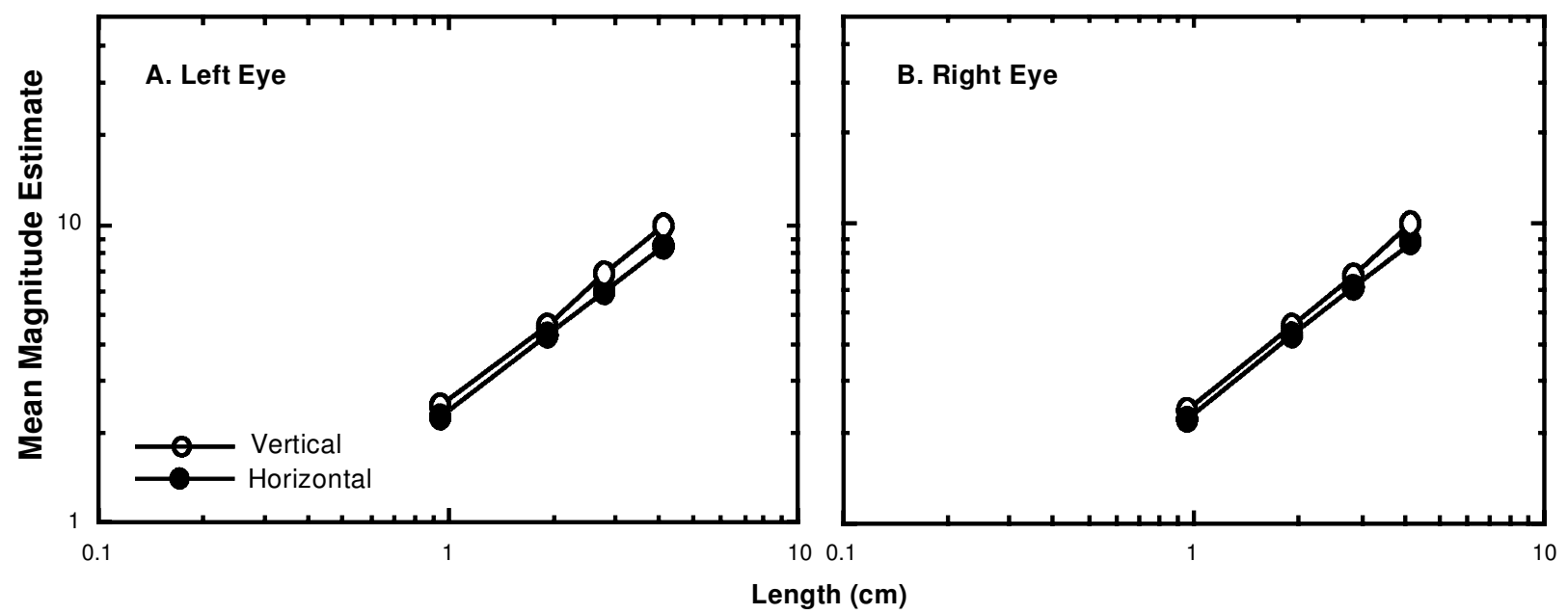

Figure 2. Baseline measure of the horizontal-vertical illusion at the left eye and at the right eye (Experiment $1 \mathrm{~A})$. Geometric average magnitude estimates of line length of horizontal lines (filled circles) and vertical lines (open circles) are plotted against physical length.

eye. To this end, we created two contextual conditions by using different combinations of horizontal and vertical lengths. The first set (Context A) comprised relatively long verticals and short horizontals, whereas the second set (Context B) comprised relatively long horizontals and short verticals. Following Armstrong and Marks (1997), we expected that exposure to Contextual Set A would cause the length of the verticals to be underestimated relative to baseline, thereby shrinking the HVI, whereas exposure to Contextual Set B would cause the length of the horizontals to be underestimated relative to baseline, thereby enlarging the HVI. Following exposure of one eye to the contextual stimulus set, we tested for contextual aftereffects in both eyes. If DCEs are not specific to the eye in which they are induced, the size of the HVI in the unexposed (contralateral) eye should be contingent on the contextual manipulation at the exposed (ipsilateral) eye. That is, the HVI in the contralateral eye should decline in size when the ipsilateral eye is exposed to Context $\mathrm{A}$ and should increase when the ipsilateral eye is exposed to Context B. Alternatively, DCEs may be specific to individual eyes, in which case the size of the HVI in the contralateral eye should stay at the baseline level.

\section{Method}

Participants. Twelve participants, 4 men and 8 women, were paid to serve in Experiment 1B. None had participated in Experiment 1A.

Stimuli and Procedure. The apparatus and procedure were like those of Experiment 1A, except for the following changes. Two contextual stimulus sets were constructed: Context A comprised relatively long vertical lines $(1.9,2.85,4.12$, and $6.0 \mathrm{~cm})$ and relatively short horizontal lines $(0.65,0.953,1.9$, and $2.85 \mathrm{~cm})$, with the two shortest verticals and two longest horizontals common to both sets. Context B comprised relatively long horizontal lines (1.9, $2.85,4.12$, and $6.0 \mathrm{~cm})$ and relatively short vertical lines $(0.65$, $0.953,1.9$, and $2.85 \mathrm{~cm}$ ), with the two shortest horizontals and two longest verticals common to both sets. Each participant viewed the context-inducing stimuli with only one eye-the left eye in 6 participants and the right eye in the other 6 . To avoid carryover effects, the two contextual conditions were presented in different sessions separated by at least $24 \mathrm{~h}$. Order of presentation of the contextual conditions was counterbalanced across participants.

Each session began with 10 practice trials, after which the contextual stimuli were presented to the chosen eye. Each line was presented six times in all, making 64 randomly ordered test trials at the context induction stage. Subsequently, we measured the HVI in both eyes by presenting the baseline stimulus set used in Experiment $1 \mathrm{~A}$. Each line was presented twice in all to each eye, making 32 presentations. The sequence of contextual-condition/ test-condition was given four times, so the total number of experimental trials, excluding practice, was 384. A typical session lasted about $25 \mathrm{~min}$.

\section{Results and Discussion}

Judgments of contextual stimuli. Figure 3 shows the average judgments for Contextual Conditions A and B collapsed across the two eyes. Note that the two contextual conditions exerted differential effects on the magnitude of the HVI. The vertical lines were judged to be only slightly longer than the horizontal lines in Contextual Condition A, resulting in an average HVI of $4.3 \%$, but much longer in Condition B, resulting in an average HVI of $17.2 \%$. As compared with the average size of the HVI obtained at baseline in Experiment 1 A (11.45\%), the effects of the two contextual conditions were similar in magnitude but opposite in direction. The size of the HVI was reduced by $7.15 \%$ [a significant difference; $t(22)=2.68, p<.05]$ under Contextual Condition A and was enlarged by $5.75 \%$ under Contextual Condition B [again, a significant difference; $t(22)=2.38, p<.05$ ]

We entered the log-transformed data obtained at the two common lengths at each orientation into an ANOVA, using contextual condition (A, B), orientation (horizontal, vertical), and length as within-subjects variables and exposed eye (left, right) as a between-subjects variable. As was expected, the main effects of length and orienta- 


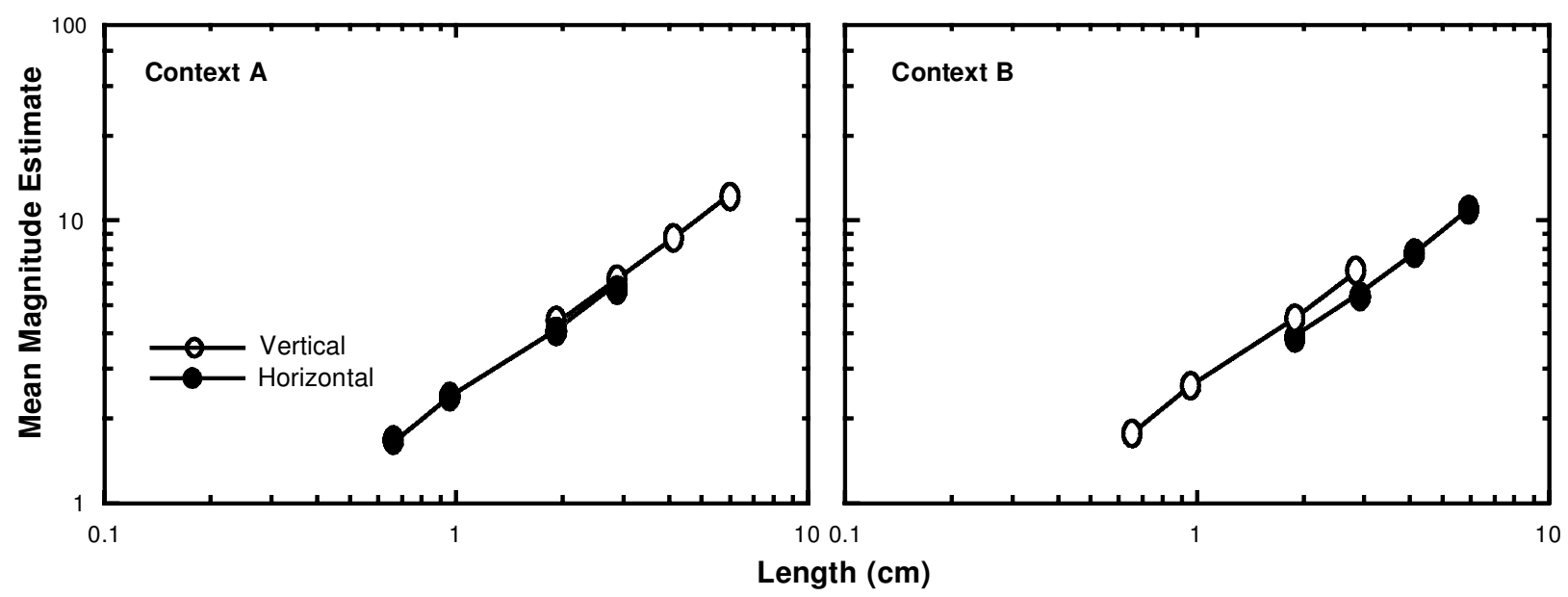

Figure 3. Geometric average magnitude estimates of line length of horizontal lines (filled circles) and vertical lines (open circles) under Contextual Conditions A and B, plotted against physical length (Experiment 1B). In Context A, vertical lines were relatively longer, whereas in Context $B$, horizontal lines were relatively longer.

tion were highly significant $[F(1,10)=132.9, p<.001$, and $F(1,10)=30.7, p<.001$, respectively]. The only other significant term was the interaction between orientation and contextual condition, indicating that the size of the HVI was significantly different under the two contextual conditions $[F(1,10)=27.4, p<.001]$. Next, we examined whether the DCE observed in the judgments of the context-inducing stimuli transferred to the contralateral eye.

Interocular transfer of the DCE. Figure 4 gives the average judgments for the test stimuli at the exposed eye (ipsilateral) and the unexposed eye (contralateral) following the two contextual conditions. Note first that the size of the HVI in the ipsilateral eye varies with prior context, averaging $5.48 \%$ following exposure to Contextual Condition $\mathrm{A}$ and $17.0 \%$ following exposure to Contextual Condition B. On the other hand, the effects of contextual preexposure at the contralateral eye were much less profound. Following exposure to Contextual Condition A, the average HVI at the contralateral eye was $8.7 \%$, and following exposure to Contextual Condition B, it was $12.07 \%$. It is clear that context affected the size of the HVI much more in the ipsilateral eye than in the contralateral eye.

To evaluate these effects statistically, we performed an ANOVA on the log-transformed judgments, with contextual condition (A, B), relative site of context induction (ipsilateral, contralateral), orientation (horizontal, vertical), and length as within-subjects variables. The most important finding was the significant interaction among contextual condition, relative site of context induction, and orientation $[F(1,11)=6.21, p<.05]$. That is, the effect on the HVI of varying the previous contextual condition depended on whether the contextual stimuli were presented to the ipsilateral or the contralateral eye, the ipsilateral effects being larger than the contralateral effects. In fact, the sizes of the HVI at the contralateral eye obtained under Contextual Conditions A and B (8.7\% and $12.07 \%$, respectively) were not reliably different from the HVI obtained under the baseline condition $[11.45 \% ; t(22)=1.16, p>.1$, and $t(22)=0.29, p>.5$, respectively]. Although lacking statistical significance, the size of the HVI in the contralateral eye did slightly change in the expected direction, decreasing under Contextual Condition $\mathrm{A}$ and increasing under Contextual Condition B. To round up the analysis, we note that the size of the HVI obtained at the ipsilateral eye was statistically different from baseline $[t(22)=2.23, p<.05$, for Contextual Condition A, and $t(22)=2.24, p<.05$, for Contextual Condition B]. So the effects of context did carry forward to the test stimuli presented in the same eye, but did so only very slightly, at most, to the contralateral eye.

The novel finding of Experiment 1B was that the DCE induced in one eye showed virtually no transfer to the other eye. Preexposing one eye to a contextual stimulus set had a profound influence on the perceived line length at the exposed eye, but only a weak effect, at most, on the perceived line length at the unexposed eye. It is important to note that although the size of the HVI at the unexposed eye did not differ statistically from baseline, it did change in the predicted direction, suggesting at least the possibility of partial transfer of the DCE. The strong eye-specificity of the DCE would be consistent with an early perceptual origin. However, the hint of partial transfer implies the possible presence of a central pooling mechanism that generalizes, to a small extent, changes in responsiveness between the two eyes, although it may not play a role in the actual production of the DCE.

\section{EXPERIMENT 2 Transfer of the DCE Across the Retina}

In Experiment 2, we explored further the newly found spatial specificity of the DCE. In particular, we asked 


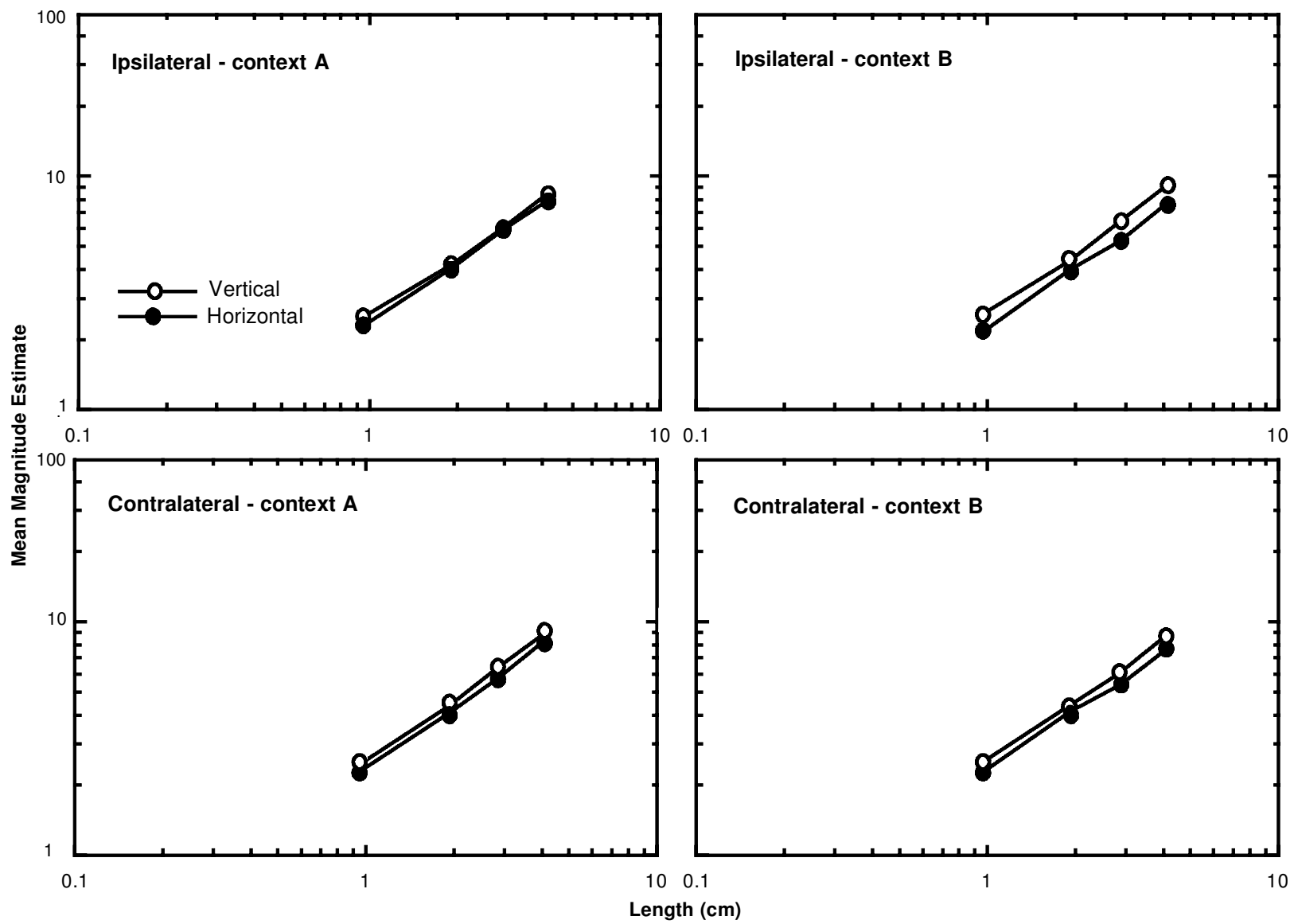

Figure 4. The effects of exposure to Contexts A and B on the test stimuli (Experiment 1B). Magnitude estimates of line length of horizontal lines (filled circles) and vertical lines (open circles) after exposure to Contextual Conditions A and B at the exposed eye (ipsilateral) and at the unexposed eye (contralateral) are plotted against physical length.

whether DCEs induced in one quadrant of the retina, or visual space, would transfer to the other three quadrants. To this end, we used the same strategy as that in Experiment 1B. First, we exposed one quadrant exclusively to one or the other of the two contextual conditions (A or B); second, we tested for aftereffects on perceived line length (i.e., the size of the HVI) in all four quadrants. To the extent that DCEs are not specific to retinal locations, exposing one quadrant to different contex tual conditions should significantly affect relative responsiveness in adjacent quadrants. In contrast, if DCEs are specific to retinal locations, aftereffects should be restricted to the exposed quadrant.

\section{Method}

Participants. Forty-eight participants, 20 men and 28 women, were paid to serve. None had served in the previous experiments. The participants were randomly assigned to one of four experimental groups defined by the quadrant selected for contextual exposure. The contextual stimulus set could be presented to the high right (HR), high left (HL), low right (LR), or low left (LL) quadrant. Each participant served in only one experimental group.

Apparatus. Booth, computer, and screen arrangements were like those used in Experiments $1 \mathrm{~A}$ and $1 \mathrm{~B}$, except that no divider was used to split the participant's visual field. Viewing distance was approximately $40 \mathrm{~cm}$ and was controlled by a fixed head/chin-rest.

Stimuli and Procedure. Each participant served in two sessions, receiving either Contextual Condition A or Contextual Condition $\mathrm{B}$ in a given session. The sessions were separated by at least $24 \mathrm{~h}$, and their order was counterbalanced. Each session contained the following sequence of events. First, a baseline measurement of the HVI was taken in all four quadrants, using the horizontal and vertical lines from Experiment $1 \mathrm{~A}$. To this end, the aperture at the middle of the screen was divided into four quadrants of equal size. The location of each line on the screen was defined by its center, which always fell on the midpoint of one of the quadrants. Each line was presented twice at each quadrant, making 64 baseline trials. Second, Contextual Stimulus Set A or B, identical to those of Experiment $1 \mathrm{~B}$, was presented to just one of the four quadrants. Each line was presented eight times in all, making 64 context-inducing trials. Finally, we tested for aftereffects of contextual stimulation at each of the four quadrants. Again, the test stimuli were identical to the baseline stimuli, but now each line was presented only once at each quadrant, making 32 test trials. The entire cycle of contextinducing trials + test trials was presented three times in all, making 352 line-judgment trials in each session [64 baseline trials $+(64$ context trials +32 test trials) $* 3$ ].

In order to limit the contextual stimuli to only one retinal quadrant, it is imperative to control for the participants' eye movements during stimulus presentations. To this end, we deployed three dif- 
ferent strategies. First, lines were presented for a short duration of $60 \mathrm{msec}$, presumably shorter than the initiation of eye movements. Second, just before each stimulus appeared, the fixation point changed its color from black to red, thereby exogenously capturing the participant's attention. Third, randomly interspersed among trials containing lines were 88 catch trials in which a letter $\mathrm{C}, \mathrm{O}, \mathrm{P}$, or $\mathrm{Q}$ appeared for $60 \mathrm{msec}$ at the fixation point. On these trials, the participants were instructed to report the letters by typing them. Data from those participants who failed to report the letters at $90 \%$ accuracy during either the first or the second session were excluded from the analysis. Surprisingly, our criteria proved too stringent for 25 individuals, who failed substantially to meet the criterion of $90 \%$ accuracy. However, we are reasonably convinced that the 48 participants who did meet our criteria did fixate on the middle of the screen throughout both experimental sessions. Adding the 88 catch trials and 10 practice trials at the beginning of each session to the 352 main trials made a total of 450 . A typical experimental session lasted about 30-35 min.

\section{Results and Discussion}

Baseline measurements. Our first analysis evaluated the HVI at baseline in each of the quadrants. Baseline judgments were collapsed across the two contextual sessions and were entered into an ANOVA, with quadrant (HL, HR, LL, and LR), orientation (horizontal, vertical), and length as within-subjects variables and experimental group as a between-subjects variable. Only two terms reached statistical significance: length $[F(3,132)=$ $865.7, p<.001]$ and orientation $[F(1,44)=290.5, p<$ $.001]$, the latter indicating the presence of the HVI. More important, as we anticipated, the size of the HVI did not differ across the four quadrants $(25.3 \%$ for HL, $26.1 \%$ for HR, $25.6 \%$ for LL, and $24.3 \%$ for LR) or across the four experimental groups. The overall size of the HVI at baseline was $25.3 \%$, substantially larger than the $11.45 \%$ obtained in Experiment 1 $\mathrm{A}$ and larger than the typical HVI size, although still within the range reported by some studies that have used magnitude estimation (Potts, 1991; Verrillo \& Irvin, 1979). The difference between Experiments $1 \mathrm{~A}$ and 2 in the size of the HVI at baseline might reside in the difference between viewing conditions and duration of stimulus exposure. In Experiment $1 \mathrm{~A}$ the lines were presented at the fixation point for a relatively long duration, whereas in Experiment 2 the lines were presented very briefly and outside the participants' fixation. However, this difference does not compromise subsequent comparisons across the different conditions within Experiment 2.

Effects of the contextual stimuli. The effects of Contextual Conditions A and B on the size of the HVI at the

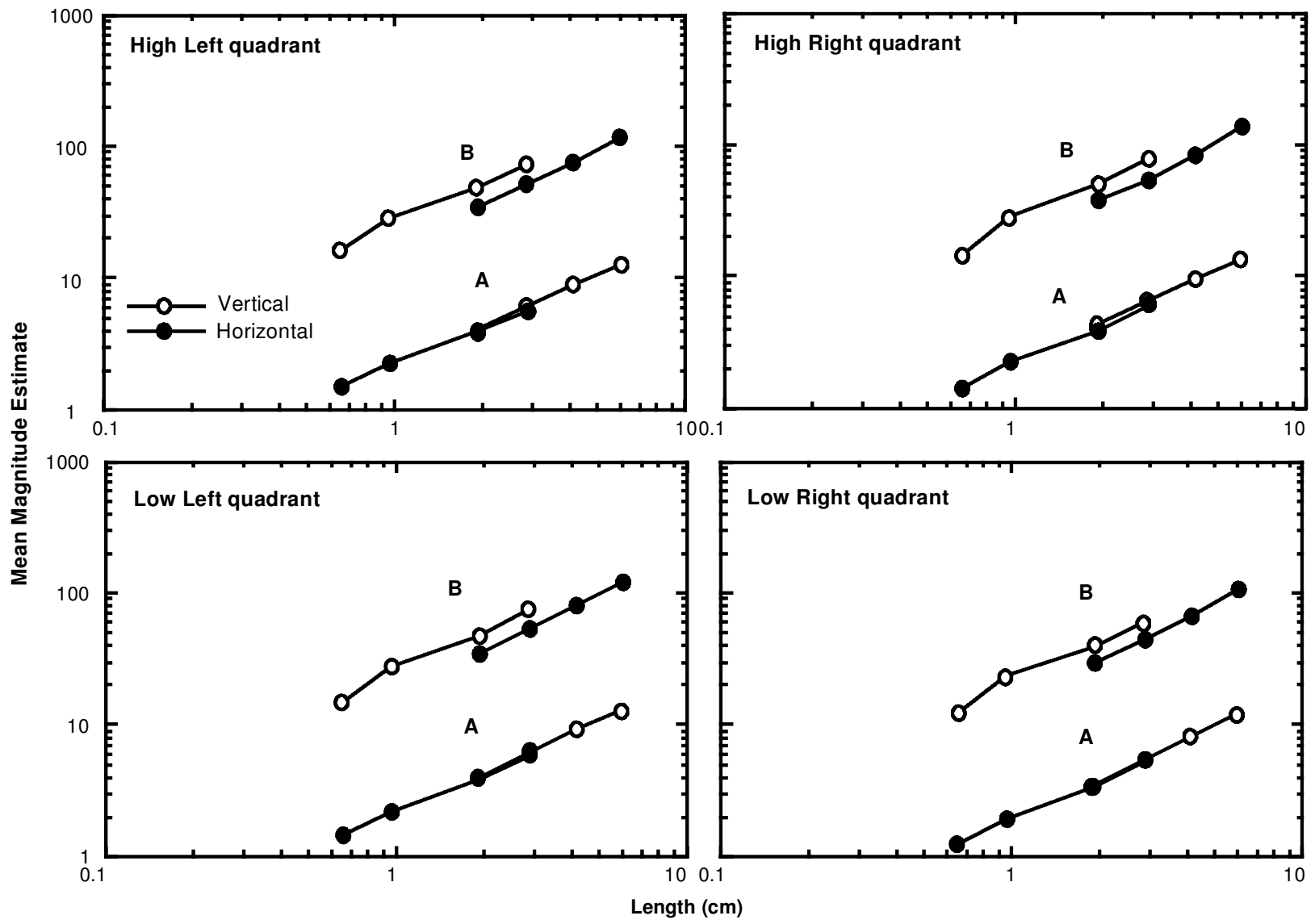

Figure 5. Estimates of line length of horizontal lines (filled circles) and vertical lines (open circles) under Contextual Conditions A and $B$ at the four quadrants, plotted against physical length (Experiment 2). Each quadrant represents a different experimental group (for clarity, length judgments of Context $B$ are multiplied by 10). 

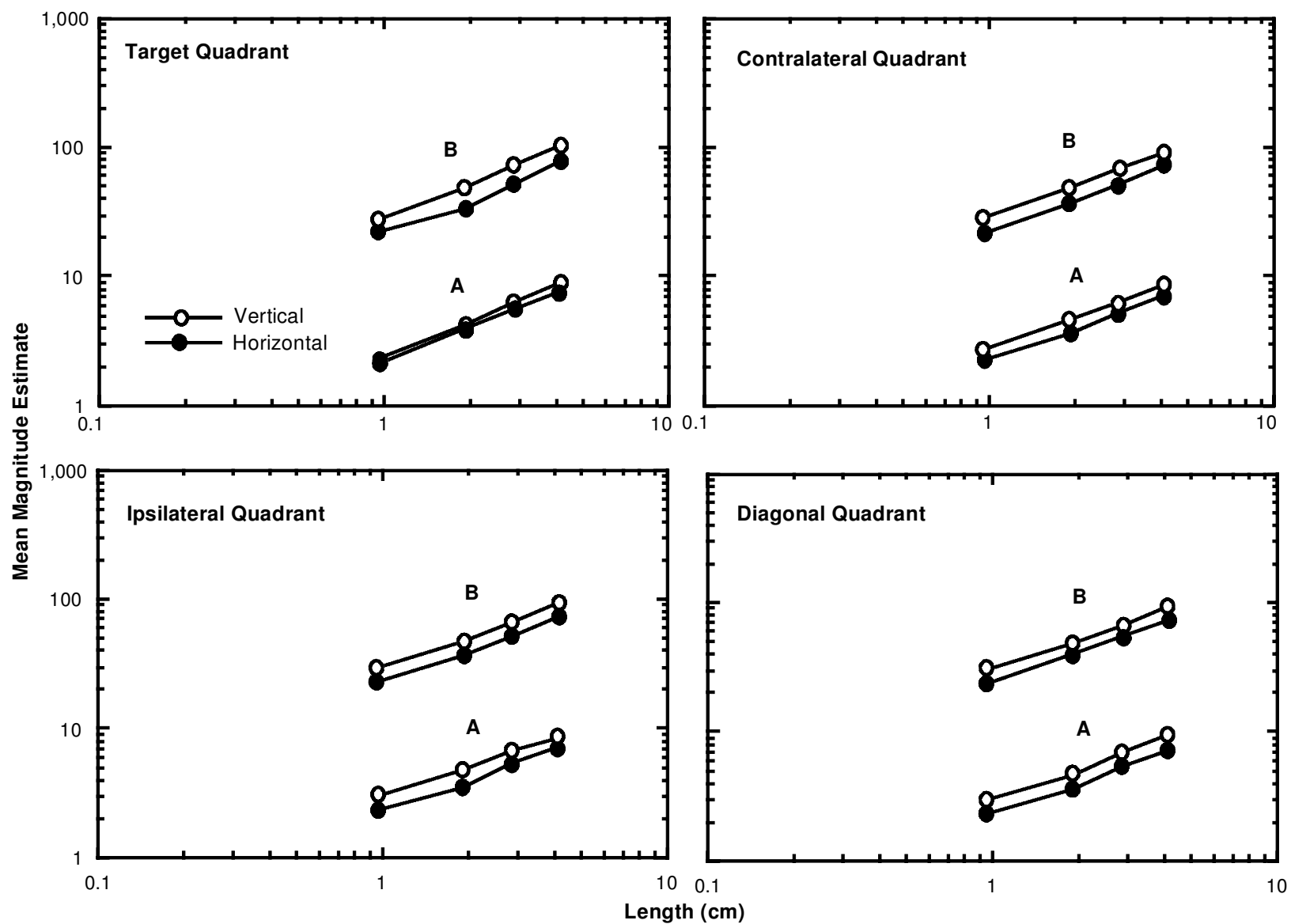

Figure 6. The effect of exposure to Contexts A and B on the test stimuli (Experiment 2). Estimates of the line length of horizontal lines (filled circles) and vertical lines (open circles) after exposure to Contextual Conditions A and B at the exposed quadrant (target) and at the adjacent quadrants (contralateral, ipsilateral, and diagonal) are plotted against physical length (for clarity, length judgments of Context $B$ are multiplied by 10$)$.

four quadrants are presented in Figure 5. As was expected, the HVI was smaller in Contextual Condition A than in Contextual Condition B. Furthermore, this modulation of the HVI's size was apparent at all four quadrants.

We entered the logarithmically transformed judgments obtained for the two common lengths at each orientation into an ANOVA, using contextual condition (A, B), orientation (horizontal, vertical), and length as within-subjects variables and context-exposed quadrant (HL, HR, LL, LR) as a between-subjects variable. As was expected, the main effects of length and orientation were highly significant $[F(1,46)=113.5, p<.001$, and $F(1,46)=31.4, p<$ .001 , respectively]. More important, the interaction between orientation and contextual condition was also significant $[F(1,46)=31.0, p<.001]$. Thus, the size of the HVI differed reliably under the two contextual conditions, confirming our visual inspection of the data. The lack of interaction between the exposed quadrant and orientation $(F<1)$ indicates that the two contextual conditions exerted similar effects on perceived length regard- less of the quadrant of contextual exposure. Thus, the data obtained in the contextual conditions can be collapsed across all four quadrants. The overall HVI under Contextual Conditions A and B was $6.2 \%$ and $38.0 \%$, respectively. Apparently, Contextual Condition A had a greater effect on the size of the HVI than did Contextual Condition B. The size of the HVI was reduced by $19.1 \%$ (from $25.3 \%$ at baseline) under Contextual Condition A but increased by only $12.7 \%$ under Contextual Condition B. However, comparing both the reduction in the HVI obtained under Contextual Condition $\mathrm{A}$ and the inflation in its size obtained under Contextual Condition B with the size of the HVI at baseline reveals both changes to be statistically significant $[F(1,47)=96.6, p<.01$, and $F(1,47)=$ $38.5, p<.01$, respectively]. Thus, despite the asymmetry, our contextual manipulation was successful: We managed to modulate the size of the HVI in different quadrants by exposing them to stimulus sets containing horizontal and vertical lines differing in relative length. Furthermore, the effects of Contextual Conditions A and B were similar across the four quadrants-shrinking the size of the 
HVI under the former and increasing it under the latter. Next, we examined whether the observed contextual effects transferred among quadrants.

Transfer of the DCE across the retina. We categorized each quadrant according to its relative position as follows: (1) target, the quadrant that received the contextual manipulation; (2) ipsilateral, the quadrant at the same side of the visual field as the target but above or below it; (3) contralateral, the quadrant on the opposite side of the visual field but at the same height as target; and (4) diagonal, the quadrant on the opposite side of the visual field and at a different height. Next, we collapsed the data from the four experimental groups within these categories. Clearly, this categorization assumes that what matters is the position relative to the target.

Figure 6 shows that the effects of preexposure to Contextual Conditions A and B are evident primarily at the target quadrant itself, and not at the other quadrants. Indeed, only at the target quadrant did the size of the HVI visibly vary with the type of contextual condition. Thus, the HVI is greater under Contextual Condition B than under Contextual Condition A. These clear effects at the target quadrant did not appear to transfer substantially to the other quadrants, where the HVI looks similar under Contextual Conditions A and B.

To test these impressions statistically, an omnibus ANOVA was performed, with contextual condition (A, B), relative position in the quadrant (target, ipsilateral, contralateral, and diagonal), orientation (horizontal, vertical), and length as within-subjects variables and experimental group (context induced in HR, HL, LL, or LR) as a between-subjects variable. As was expected, of the main effects only line length and orientation were significant $[F(3,132)=1,077.4, p<.001$, and $F(1,44)=$ $441.8, p<.001$, respectively]. More important, the inter- action among contextual condition, relative position in the quadrant, and orientation was significant $[F(3,132)=$ $18.5, p<.001]$, confirming the quadrant-dependent effects on the HVI of contextual preexposure. On the basis of a visual inspection of the data, the effects appear largely restricted to the target quadrant. Indeed, the interaction between contextual condition and orientation was significant only at the target quadrant $[F(1,47)=64.1$, $p<.01$, for the target quadrant], and not at any other quadrant $[F(1,47)=2.1, p>.05$, for the contralateral quadrant; $F(1,47)=1.9, p>.05$, for the ipsilateral quadrant; $F<1$ for the diagonal quadrant].

To test this interpretation further, we computed the size of the HVI (in percentages) for the two contextual conditions at each of the relative positions. The results appear in Figure 7. Note that the largest disparity between the effects of Contextual Conditions A and B on the size of the HVI was registered at the target quadrant, where the HVI was $10.5 \%$ following Context $\mathrm{A}$ and $34.4 \%$ following Context B, a difference of $23.9 \%$. By contrast, the smallest disparity was registered at the diagonal quadrant, the location most distant from the target, where the HVI was $25.3 \%$ following Condition A and $25.7 \%$ following Condition B, a trivial difference of only $0.4 \%$. Differential effects of the two contextual conditions at the contralateral and ipsilateral quadrants were not impressive either, being $6.9 \%$ for the former $(21.6 \%$ following Condition A vs. 28.5\% following B) and 3.7\% for the latter (24.3\% following A and $28 \%$ following B). We performed an ANOVA on these differences scores. As was expected, the main effect of relative location was highly significant $[F(3,47)=18.16, p<.001]$. Subsequent contrasts revealed that only the target quadrant differed significantly from the other three quadrants (which did not differ among themselves).

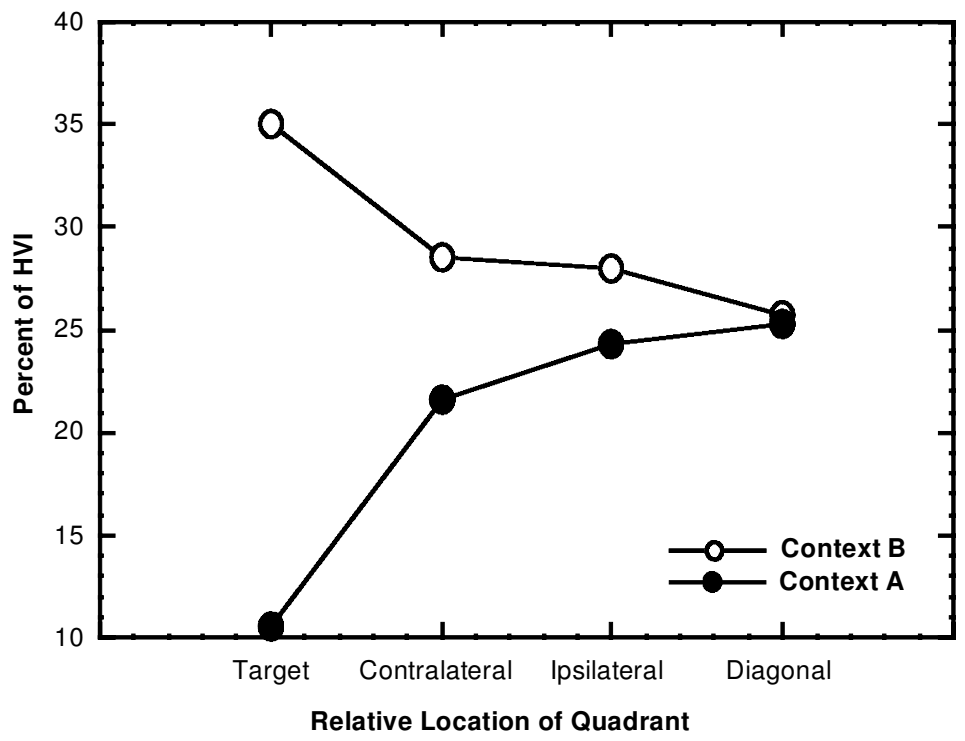

Figure 7. Average percentage of the HVI under Contextual Conditions A and $B$, plotted against relative quadrant position. 
Another way to assess the magnitude of the DCE at the different quadrants is to compare the magnitude of the HVI with our baseline index. For both contextual conditions, only in the target quadrant did the magnitude of the HVI differ significantly from baseline $[F(1,47)=$ $39.0, p<.01$, and $F(1,47)=11.1, p<.01$, respectively, for Contextual Conditions A and B]. Thus, we conclude that the DCEs induced at the target quadrant transferred poorly to the other three quadrants. Exposure to contextual sets of horizontal and vertical lines differing in relative length modulated the size of the HVI in the region of exposure. There was, however, a small-albeit, statistically unreliable - amount of transfer in the expected direction, especially in the contralateral and the ipsilateral quadrants; in the diagonal quadrant, the HVI was virtually identical to its size at baseline. It appears that the differential effects dissipated with an increase in distance from the point of induction.

The present analyses support the conclusion that the DCEs did not transfer in a significant manner from one retinal quadrant to the others. Note that we took great care in this experiment to control for eye movements. The stimuli were presented rapidly, and the participants were engaged in a secondary task at the fixation point to help ensure that the stimuli would fall on the designated regions of the retina. But the stimuli presented at each location on the retina and, thus, at each location in the visual field also appeared at a corresponding unique location in external space-in this case, the same location on the computer screen. In other words, location on the retina covaried perfectly with location in the visual world.

Are the DCEs revealed in Experiment 2 specific to different parts of the retina, or are they specific to different parts of external space? The former would imply that DCEs arise at a relatively early, retinotopic level in the visual system, whereas the former would imply that DCEs arise at a relatively late level of processing, defined by locations in space. Perhaps DCEs are specific to objects in the visual world, rather than to stimulation in the visual field. Location in external space can, for example, affect the HVI. When people judge horizontal and vertical extents with their visual fields tilted $90^{\circ}$ (e.g., lying on their sides), a mismatch is created between the location of the objects in the visual field and their retinal projection, a condition that nearly eliminates the HVI (Higashiyama, 1996). It is conceivable that ecological factors also influence orientation-specific DCEs. At present, there is little pertinent evidence. Marks and Armstrong (1996) reported evidence that DCEs may not be specific to stimulus objects in space. They found that orientation-specific DCEs in perceived length induced visually and haptically by a single common set of stimulus objects were wholly independent. Nevertheless, those cross-modal findings are neither conclusive nor exhaustive. In Experiment 3, we sought to determine whether visual DCEs are specific to stimulation of regions of the retina (visual field) or to regions of external visual space.

\section{EXPERIMENT 3}

Experiments 1 and 2 used a paradigm involving baseline stimulation, induction of context, and then test stimulation. This design relies on detecting aftereffects of the contextual influence induced in the test phase. Since DCEs have been found to dissipate rather quickly (Marks, 1992a), their magnitude can be underestimated in the test phase. Thus, for Experiment 3, we chose an even more powerful design, one that combined induction of DCEs and testing of them in the same block of trials. Within a given session, Contextual Stimulus Sets A and $B$ were presented at two different spatial locations on the computer screen. At the beginning of each trial, we cued the location at which the line would appear. The participants were instructed to look directly at the fixation point and subsequently at the stimulus while assessing its length. Thus, the lines always fell on the same part of the retina (presumably, across the fovea), but each contextual set of stimuli had a different location in external space (readily discernible given the level of illumination of the screen's background).

Note that Contextual Set A and Contextual Set B are complementary with regard to the distribution of lengths. Contextual Set A comprised long verticals and short horizontals, whereas Set B comprised long horizontals and short verticals. Overall, then, the means and the ranges of horizontal lengths and vertical lengths were the same in each contextual condition. So if DCEs are specific to retinal location, then because all of the stimuli were presented to the fovea, the two contextual sets should merge functionally, and the size of the HVI measured at the two context-defined locations should equal the size of the HVI obtained at baseline. Alternatively, if DCEs are specific to location in visual space, each contextual set should exert an independent effect and, thus, appropriately modify the size of the HVI at each location. In this case, relative to baseline, the HVI should decrease at the location of Context $\mathrm{A}$ and increase at the location of Context B.

\section{Method}

Participants. Twelve participants, 6 men and 6 women, were paid to serve in Experiment 3. None had participated in the previous experiments.

Stimuli and Procedure. The apparatus, stimulus sets, and procedure were like those of Experiment $1 \mathrm{~A}$ and Experiment 1B, except for the following changes. No divider split the visual field. Each trial began with presentation of a fixation point located halfway between the center of the screen and either the left or the right border. This was followed, after a 500-msec delay, by a 1,000msec presentation of the line, centered on the location of the fixation point. The participants were instructed to move their eyes, but not their heads, to the location of the fixation point, and then, after the presentation of the line, were prompted to enter their judgments of its length on a keyboard. Thus, half of the stimuli fell on the left of the screen, and half fell on the right, but all fell on the central region of the retina.

After 10 practice trials, the baseline set of stimuli was presented twice at each of the two spatial locations. Next, randomly inter- 
mixed in a single block, stimuli from Contextual Set A were presented at one spatial location, and stimuli from Contextual Set B were presented at the other location. The assignment of contextual condition to one location or the other was counterbalanced among subjects. The entire set of contextual stimuli was presented a total of six times over the course of the session, making 128 experimental trials in all, excluding practice.

\section{Results and Discussion}

Judgments of the baseline stimuli. The magnitude of the HVI was $11.2 \%$ at the right spatial location and $11.3 \%$ at the left spatial location. The data (log transformed) were subjected to an ANOVA, using spatial location (left, right), orientation (horizontal, vertical), and length as within-subjects variables. Only two terms were significant: the expected main effects of length $[F(3,33)=$ $270.6, p<.001]$ and orientation $[F(1,11)=33.2, p<$ $.001]$. Importantly, there was no interaction between orientation and spatial location $(F<1)$, indicating that the size of the HVI on the right did not differ from its size on the left. Thus, we used their average $(11.25 \%)$ to assess the effect of varying the stimulus context at the two spatial locations.

Judgments of the contextual stimuli. Figure 8 shows the average judgments for Contextual Conditions $\mathrm{A}$ and B collapsed across the two spatial locations. Note that the magnitudes of the HVI obtained with the two contextual conditions are comparable. Indeed, an ANOVA using contextual condition (A, B), orientation (horizontal, vertical), and length as within-subjects variables and spatial location (left, right) as a between-subjects variable failed to show an interaction of orientation by contextual condition $(F<1)$. The HVIs in Contextual Conditions $\mathrm{A}$ and $\mathrm{B}$ were $12.3 \%$ and $10.2 \%$, respectively.
The spatial location of the contextual stimuli did not matter, since location did not interact either with orientation $(F<1)$ or with orientation $\times$ contextual condition $[F(1,10)=4.63, p>.05]$. The magnitudes of the HVI obtained in Contextual Conditions A and B and at baseline were all similar in size (indeed, the very small numerical difference between the magnitudes of the HVI in Conditions A and B goes opposite to the expected direction). An ANOVA, with experimental condition (baseline, A, B) and orientation (horizontal, vertical) as within-subjects variables, revealed no interaction between experimental condition and orientation $(F<1)$.

These results strongly suggest that the two (complementary) contextual sets of stimuli, A and B, merged functionally into a single neutral set whose effect was essentially equivalent to that of the baseline set. This happens when the effects of the contextual set at one location in external space pool with the effects of the other contextual set at another location. Equivalently, we may say that the contextual effect produced at each location in external space transfers to the other location; and because the contextual sets of stimuli presented to the two spatial locations are complementary, the DCEs cancel. Taken together with the results of the Experiment 2, the present results lead us to conclude that the key ingredient to the spatial specificity of these DCEs is the location of the stimuli on the retina, rather than their location in external space.

\section{GENERAL DISCUSSION}

Three experiments tested the spatial specificity of DCEs in judgments of line length. We used two contex-

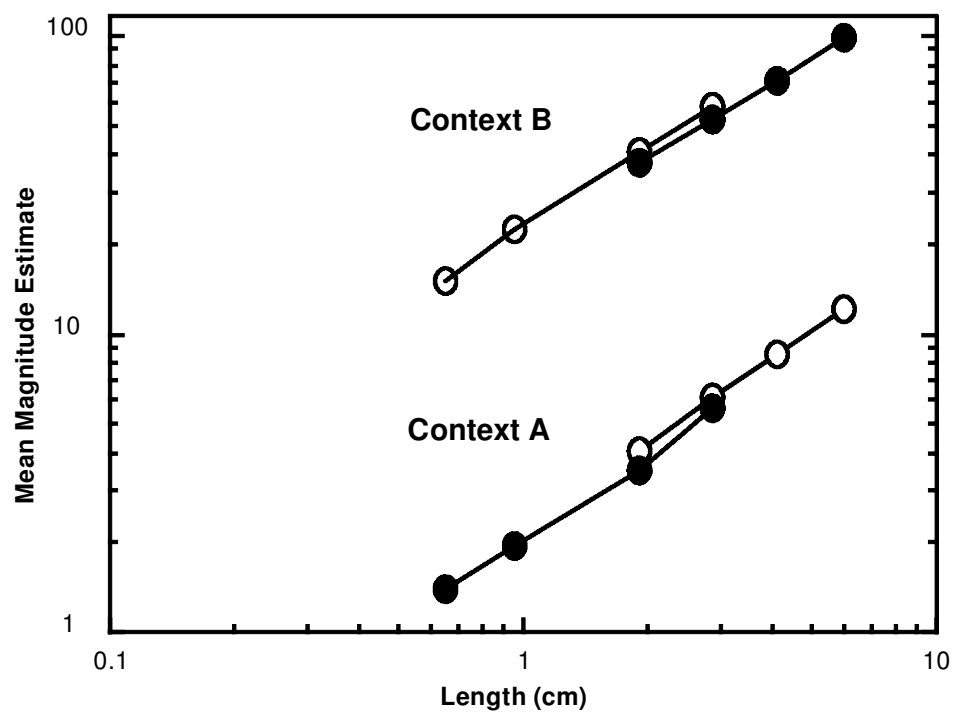

Figure 8. The effect of simultaneous exposure to Contexts A and B presented at different locations in the visual field (Experiment 3). Estimates of the line length of horizontal lines (filled circles) and vertical lines (open circles) are plotted against physical length (for clarity, length judgments of Context $B$ are multiplied by 10). 
tual sets comprising horizontal and vertical lines with different relative lengths. One contextual set contained relatively long verticals and short horizontals, and the other contained relatively long horizontals and short verticals. The former set is associated with a decrease in the size of the HVI, and the latter is associated with an increase in its size (Armstrong \& Marks, 1997). Experiment 1 showed that exposing one eye to different contextual sets had virtually no effect on the size of the HVI in the other eye. Experiment 2 showed that exposing one quadrant of the retina to different contextual sets of stimuli had little effect on the size of the HVI at the other three quadrants. Finally, Experiment 3 confirmed that it was the location on the retina, rather than the location in external visual space, that was responsible for the spatial specificity uncovered in Experiment 2. Thus, DCEs appear to be largely specific to the stimulated eye and to the region of the retina to which they are presented.

The results did suggest the possibility of a small amount of interocular and intrafield transfer of the DCE in the predicted direction, reminiscent of Marks's (1996) finding of partial interaural transfer of DCEs for loudness, although transfer in the auditory system was more substantial. These traces of visual transfer point to the possible involvement of some central pooling mechanism that spatially generalizes changes in sensitivity across different parts of the visual field. However, the meager amounts of spatial transfer suggest that this central mechanism may play a modest role, at most, in the actual production of the DCEs; the strong spatial specificity of the DCEs uncovered in this study implies instead that they originate in a relatively early stage of visual processing.

Further insight into the locus of DCEs may be gained by considering their possible relation to the McCollough effect (McCollough, 1965). The McCollough effect is an orientation-specific color aftereffect. For example, viewing in alternation a horizontal red-and-black grating and a vertical green-and-black grating induces the white bars of a horizontal black-and-white grating to appear tinged with green and the white bars of a vertical black-andwhite grating to appear tinged with pink. Several empirical similarities between the McCollough effect and the DCEs make their comparison fruitful.

First, both phenomena represent perceptual interactions between purportedly independent perceptual dimensions. The McCollough effect shows how orientation can affect the perception of color, whereas the DCEs show how orientation can affect the perception of length. Second, the McCollough effect, like DCEs, shows strong spatial specificity: It does not transfer between the two eyes (Murch, 1972) or from one portion of the visual field to another (Stromeyer, 1972).

These findings, along with several others (for a review, see Humphrey, 1998), have been taken to support the claim that the McCollough effect reflects adaptation in orientation-specific color-coding detectors located relatively early in the visual pathway, perhaps as early as V1 (but see Siegel, Allan, \& Eissenberg, 1992, for another explanation). Given their empirical affinity to the McCollough effect, it is reasonable to consider the possibility that DCEs may reflect some kind of adaptation in orientation-specific length detectors, being induced before the point of binocular and retinal convergence (or perhaps later, in an independent monocular channel), conceivably in the primary visual cortex, where monocular orientation-specific length detectors can be found (Hubel $\&$ Wiesel, 1968, 1979). On the other hand, McCollough effects are well known to be long lasting, whereas in hearing, at least, DCEs seem to dissipate fairly quickly (Marks, 1992a).

Throughout this study, we used the method of magnitude estimation to gauge the perception of line length. To be sure, assigning numbers to stimulus magnitude requires high-level processing and is subject to various decisional biases that may influence the eventual response (e.g., Marks \& Algom, 1998; S. S. Stevens, 1975). But it is important to note that DCEs have been demonstrated using a variety of methods, including ones that do not rely on quantitative ratings of magnitude and, thus, are less vulnerable to such biases. For example, Armstrong and Marks (1997) used the method of paired comparison, asking their participants to compare directly the lengths of horizontal and vertical lines. Results showed HVIs and DCEs that were comparable in magnitude to those reported here in Experiments 1 and 3. Marks (1992a, 1994) used a similar paired-comparison procedure, finding DCEs in loudness perception similar to those previously obtained by magnitude estimation (Marks, 1988). Marks (1993, 1994) and Mapes-Riordan and Yost (1999) used a similar method of direct comparison of loudness and reported comparable findings. And Marks (1996) used direct loudness comparison to measure the interaural transfer of auditory DCEs. All of the evidence at hand suggests that DCEs are generally independent of the method of psychophysical measurement. In fact, Marks (1992a, 1994) found DCEs for loudness measured by paired comparison to be slightly greater in size than DCEs measured by magnitude estimation, a difference hypothesized to reflect greater differential adaptation when signals activating different perceptual channels are presented in close proximity in the same trial. In any case, there is no reason to attribute the presence or absence of DCEs observed in the present study to the method of magnitude estimation.

Although the present findings may not rule out the possibility that DCEs originate relatively late in visual processing, the findings do not square well with a model postulating that they arise from central decisional processes. If exposure to relatively long horizontal or vertical lines causes nothing more than a general shift in the criteria for judging length, we would expect this shift to be manifested equally across the visual field. But this did not happen, at least not in significant amounts. Rather, our results appear to be more in tune with the hypothesis 
that the DCEs reflect a change in responsiveness in local, functionally (for the most part) monocular, albeit probably central, visual channels encoding horizontal and vertical extents.

These results also bear phenomenal affinity to the well-documented phenomena of perceptual learning. The honing of one's performance on tasks such as texture discrimination (Karni \& Sagi, 1991), orientation discrimination (Shiu \& Pashler, 1992), and vernier acuity (Poggio, Fahle, \& Edelman, 1992) is spatially specific. Often, the newly acquired skill does not transfer to a different situation in which the stimuli are presented at a new, unpracticed spatial location. Indeed, the locus of perceptual learning has been attributed to neural changes at relatively early stages of visual processing, presumably in the vicinity of the primary visual cortex (Ahissar, 1999; Goldstone, 1998). Perhaps differential context effects represent another instance of spatially dependent perceptual learning. A model of perceptual learning would presumably rely on the notion that, over the course of a test session, subjects would "learn" the contextual distribution of the stimulus levels and, in light of this, would come to adjust their responses accordingly, thereby producing a DCE. We believe, however, that the DCEs can better be conceptualized as reflecting the operation of perceptual adaptation than as a process of learning. Several properties of DCEs point in this direction.

Previous research has shown that the induction of DCEs is rapid, taking only a few trials at most (MapesRiordan \& Yost, 1999, Experiment 1; Marks, 1992a, Experiment 2). Recovery, too, can be evident just a few trials after the contextual stimuli are replaced by a "neutral" set of stimuli (Marks, 1992a); recovery appears to take a matter of several minutes. By way of contrast, perceptual leaning often requires extensive practice, and its aftereffects may linger for days, weeks, or even months (Karni \& Sagi, 1993). Furthermore, DCEs can be induced simply by presenting context-inducing stimuli, without requiring the participants to respond overtly (Marks, 1993). In addition to the evidence that fullblown DCEs arise after only a small number of trials and dissipate fairly quickly, DCEs depend much more on high than on low stimulus magnitudes, both in the perception of visual length (Armstrong \& Marks, 1997) and in the perception of loudness (Marks, 1993, 1994). In their rapid onset and dissipation and in their reliance on relatively high stimulus magnitudes, DCEs more closely resemble phenomena of adaptation than ones of perceptual learning.

Stimulus magnitude played a role in the present study too. In Experiment 2, at baseline, a vertical line received an average estimate of 5.31, and a horizontal line received an average estimate of 4.23 - an HVI of about $25 \%$. After exposure to the stimuli of Condition A, which contained relatively long verticals and short horizontals, a vertical line received an average estimate of 4.69, whereas a horizontal line still received an average estimate of 4.24 -an HVI of about $10 \%$. It appears that the large re- duction in the size of the HVI was due to underestimation of the verticals, and not to overestimation of the horizontals. Analogously, after exposure to the stimuli of Condition B, which contained relatively long horizontals and short verticals, a vertical line remained at 5.36, on average, whereas a horizontal line received an average estimate of 3.98-an HVI of about $34.4 \%$. In Condition $\mathrm{B}$, the increase in the size of the HVI was due to underestimation of the horizontals, and not to over-estimation of the verticals.

This analysis supports the contention that DCEs are largely due to some kind of attenuation-like process resulting from stimulating an orientation-specific channel at relatively high stimulus levels, as has been reported for loudness (Mapes-Riordan \& Yost, 1999; Marks, 1993, 1994). Superficially, these decrements in perceived magnitude resemble those that arise after presenting prolonged and relatively strong adapting or fatiguing stimuli. In contrast to reductions in perceived magnitude resulting from sensory adaptation or fatigue, however, DCEs arise from more moderate, transient stimulation. Thus, one need not apply excessive and prolonged stimulation to achieve a change in sensitivity of the perceptual system; mild, transient stimulation distributed differentially across perceptually independent channels can produce a similar effect.

\section{REFERENCES}

Ahissar, M. (1999). Perceptual learning. Current Directions in Psychological Science, 4, 124-128.

Armstrong, L., \& Marks, L. E. (1997). Differential effects of stimulus context on perceived length: Implications for the horizontal-vertical illusion. Perception \& Psychophysics, 59, 1200-1213.

Canevet, G., Scharf, B., \& Botte, M.-C. (1985). Simple and induced loudness adaptation. Audiology, 24, 430-436.

Cohen, J., MacWhinney, B., Flatt, M., \& Provost, J. (1993). PsyScope: An interactive graphic system for designing and controlling experiments in the psychology laboratory using Macintosh computers. Behavior Research Methods, Instruments, \& Computers, 25, 257-271.

Goldstone, R. L. (1998). Perceptual learning. Annual Review of Psychology, 49, 585-612.

Higashiyama, A. (1996). Horizontal and vertical distance perception: The discorded-orientation theory. Perception \& Psychophysics, 58, 259-270.

Hubel, D. H., \& Wiesel, T. N. (1968). Receptive fields and functional architecture in the cat's visual cortex. Journal of Physiology, 160, 106-154.

Hubel, D. H., \& Wiesel, T. N. (1979). Brain mechanisms of vision. Scientific American, 82, 84-97.

Humphrey, G. K. (1998). The McCollough effect: Misperception and reality. In V. Walsh \& J. Kulikowski (Eds.), Perceptual constancy: Why things look as they do (pp. 31-68). Cambridge: Cambridge University Press.

KARNI, A., \& SAGI, D. (1991). Where practice makes perfect in texture discrimination: Evidence for primary visual cortex plasticity. Proceedings of the National Academy of Sciences, 88, 4966-4970.

KARni, A., \& SAGI, D. (1993). The time course of learning a visual skill. Nature, 365, 250-252.

MAPES-RIORDAN, D., \& Yost, W. A. (1999). Loudness recalibration as a function of level. Journal of the Acoustical Society of America, 106, 3506-3511.

MARKs, L. E. (1988). Magnitude estimation and sensory matching. Perception \& Psychophysics, 43, 511-525. 
MArKs, L. E. (1992a). The contingency of perceptual processing: Context modifies equal-loudness relations. Psychological Science, 3, 285-291.

MARKs, L. E. (1992b). The slippery context effect in psychophysics: Intensive, extensive, and qualitative continua. Perception \& Psychophysics, 51, 187-198.

MARKS, L. E. (1993). Contextual processing of multidimensional and unidimensional auditory stimuli. Journal of Experimental Psychology: Human Perception \& Performance, 19, 227-249.

MARKS, L. E. (1994). "Recalibrating" the auditory system: The perception of loudness. Journal of Experimental Psychology: Human Perception \& Performance, 20, 382-396.

MARKS, L. E. (1996). Recalibrating the perception of loudness: Interaural transfer. Journal of the Acoustical Society of America, 100, 473 480 .

Marks, L. E., \& Algom, D. (1998). Psychophysical scaling. In M. H. Birnbaum (Ed.), Measurement, judgment, and decision making (pp. 81-178). San Diego: Academic Press.

Marks, L. E., \& Armstrong, L. (1996). Visual and haptic representations of space. In T. Inui \& J. L. McClelland (Eds.), Attention and performance XVI: Information integration in perception and communication (pp. 263-287). Cambridge, MA: MIT Press.

MARKS, L. E., \& WARner, E. (1991). Slippery context effect and critical bands. Journal of Experimental Psychology: Human Perception \& Performance, 17, 986-996.

McCollough, C. (1965). Color adaptation of edge-detectors in the human visual system. Science, 149, 1115-1116.

McPherson, D. F., \& Anderson, C. V. (1971). Relation of temporary loudness shift to temporary threshold shift. Journal of the Acoustical Society of America, 49, 1195-1202.

Meiselman, H. L. (1968). Magnitude estimations of the course of gustatory adaptation. Perception \& Psychophysics, 4, 193-196.

Murch, G. M. (1972). Binocular relationships in a size and color orientation specific after-effect. Journal of Experimental Psychology, 93, 30-34.

Poggio, T., Fahle, M., \& Edelman, S. (1992). Fast perceptual learning in visual hyperacuity. Science, 256, 1018-1021.

PotTs, B. C. (1991). The horizontal-vertical illusion: A confluence of configural, contextual, and framing factors. Unpublished doctoral dissertation, Yale University.

Prinzmetal, W., \& Gettleman, L. (1993). Vertical-horizontal illusion: One eye is better than two. Perception \& Psychophysics, 53, 81-88.

Pryor, G. T., Steinmetz, G., \& Stone, H. (1970). Changes in absolute detection threshold and in subjective intensity of suprathreshold stimuli during olfactory adaptation and recovery. Perception \& Psychophysics, 8, 331-335.

Rankin, K. M., \& MarKs, L. E. (1991). Differential context effects in taste perception. Chemical Senses, 16, 617-629.

RANKIN, K. M., \& MARKS, L. E. (1992). Effects of context on sweet and bitter tastes: Unrelated to sensitivity to PROP (6-n-propylthiouracil). Perception \& Psychophysics, 52, 479-486.

Rankin, K. M., \& MarKs, L. E. (2000). Differential context effects in chemosensation: Role of perceptual similarity and neural communality. Chemical Senses, 25, 747-759.

Scharf, B. (1983). Loudness adaptation. In J. V. Tobies \& E. D. Schubert (Eds.), Hearing research and theory (Vol. 2, pp. 1-56). New York: Academic Press.

Schneider, B., \& Parker, S. (1990). Does stimulus context affect loudness or only loudness judgments? Perception \& Psychophysics, 48, 409-418.

Shiu, L.-P., \& PAShler, H. (1992). Improvement in line orientation discrimination is retinally local but dependent on cognitive set. Perception \& Psychophysics, 52, 582-588.

Siegel, S., Allan, L. G., \& Eissenberg, T. (1992). The associative basis of contingent color aftereffects. Journal of Experimental Psychology: General, 121, 79-94.

Stevens, J. C., \& STevens, S. S. (1963). Brightness function: Effects of adaptation. Journal of the Optical Society of America, 53, 375385.

Stevens, S. S. (1975). Psychophysics: Introduction to its perceptual, neural, and social aspects. New York: Wiley.

Stromeyer, C. F., III (1972). Contour contingent color aftereffects: Retinal area specificity. American Journal of Psychology, 85, 227 235.

Verrillo, R. T., \& Irvin, G. (1979). Absolute estimation of line length as a function of orientation and contrast polarity. Sensory Processes, 3, 261-274.

Verrillo, R. T., \& Schmiedt, R. A. (1974). Vibrotactile poststimulatory threshold shift. Bulletin of the Psychonomic Society, 4, 484-486.

WARD, W. D. (1963). Auditory fatigue and masking. In J. Jerger (Ed.), Modern development in audiology (pp. 240-286). New York: Academic Press.

(Manuscript received August 9, 2000; revision accepted for publication July 12, 2001.) 